\title{
Vom Lizenziats- zum Bologna-System: Auswirkungen auf das Prüfungsgeschehen
}

\author{
Sethe, Rolf
}

Posted at the Zurich Open Repository and Archive, University of Zurich ZORA URL: https://doi.org/10.5167/uzh-92429

Book Section

Originally published at:

Sethe, Rolf (2013). Vom Lizenziats- zum Bologna-System: Auswirkungen auf das Prüfungsgeschehen. In: Brockmann, Judith; et al. Prüfen in der Rechtswissenschaft: Probleme, Praxis und Perspektiven. Baden-Baden: Nomos, 94-106. 
Rolf Sethe, Vom Lizenziats- zum Bologna-System: Auswirkungen auf das Prüfungsgeschehen, in: Brockmann/Pilniok (Hrsg.), Prüfen in der Rechtswissenschaft - Probleme, Praxis und Perspektiven, Baden-Baden 2013, S. 94-106

\section{Vom Lizenziats- zum Bologna-System: Auswirkungen auf das Prüfungsgeschehen}

Rolf Sethe

\section{Einleitung}

Während die Schweiz das Jurastudium auf das sog. Bologna-System umgestellt hat und daher das bisherige Lizenziatssystem durch einen Bachelor- und einen Masterabschluss ersetzt hat, hält Deutschland im Fach Rechtswissenschaften am Staatsexamen fest und lehnt eine Übernahme des Bologna-Modells ab. Der nachfolgende Beitrag verfolgt zwei Ziele. Zum einen soll - aus Schweizer Perspektive - am Beispiel der Zürcher Fakultät das Prüfungsgeschehen vor und nach der Umstellung untersucht werden. Zum anderen sollen diese Ergebnisse mit dem deutschen System verglichen werden.

\section{Die Diskussion um Einführung des Bologna-Systems im Fach Rechtswissenschaften}

\section{Deutschland}

Die Ausbildung deutscher Juristen orientiert sich am Leitbild des Richterberufs. Um einen der klassischen juristischen Berufe (Richter, Staatsanwalt, Rechtsanwalt, Notar) ergreifen zu können, müssen die Erste und Zweite Juristische Prüfung, also Staatsexamina, bestanden werden ( $\$ 5$ Abs. 1 DRiG). Im Sommer 1999 fassten die europäischen Bildungsminister in Bologna den Beschluss, das europäische Bildungssystem zu vereinheitlichen, zu modernisieren und die Wettbewerbsfähigkeit des Bildungsstandorts Europa zu stärken. ${ }^{1}$ Man strebte damit die Förderung der Mobilität in räumlicher wie in kultureller Hinsicht, das lebenslange Lernen, die Qualitätskontrolle und Verzahnung des europäischen Hochschulraums an. Diese Ziele sollten vor allem durch die Schaffung eines zweistufigen Systems von konsekutiven Studienabschlüssen (Bachelor und Master), die Einführung eines vergleichbaren Leistungspunktesystems (European Credit Transfer System ECTS) und die Modularisierung der Studieninhalte erreicht werden. Die Kultus-

1 The Bologna Declaration of 19 June 1999 - Joint declaration of the European Ministers of Education, http://www.ond.vlaanderen.be/hogeronderwijs/bologna/documents/MDC/BOLO GNA_DECLARATION1.pdf (alle Internetzitate abgerufen am 14.7.2012). 
ministerkonferenz beschloss am 12. Juni 2003 zehn Thesen zur Umstellung des bisherigen Systems auf die Bachelor- und Masterausbildung. ${ }^{2}$ Bachelor- und Masterabschlüsse wurden als eigenständige berufsqualifizierende Hochschulabschlüsse eingeordnet und der Bachelorabschluss sollte fortan als erster berufsqualifizierender Abschluss und damit als Regelabschluss eines Hochschulstudiums anzusehen sein. ${ }^{3}$

Daraufhin entbrannte eine rege Diskussion über die Frage, ob die Umstellung auf das neue System auch für die Studiengänge vollzogen werden sollte, die auf Staatsexamina ausgerichtet waren. ${ }^{4}$ Der deutsche Juristenfakultätentag gab sich sehr zurückhaltend und betonte mehrfach, dass die klassischen juristischen Berufe - wie bisher - nur nach Ablegen der beiden Staatsexamina offen stehen sollten. ${ }^{5}$ Der Bachelorabschluss wurde als nicht ausreichend angesehen. Man lehnte auch zahlreiche politische Initiativen (u.a. das Stuttgarter Modell, das „4-Stufen-Modell“" sowie den Vorschlag der nordrhein-westfälischen Justizministerin Müller-Pie-

210 Thesen zur Bachelor- und Masterstruktur in Deutschland, http://www.kmk.org/fileadmin/ veroeffentlichungen_beschluesse/2003/2003_06_12-10-Thesen-Bachelor-Master-in-D.pdf.

3 Dass diese Einordnung, die sicherlich auch auf dem Gedanken an Einsparungen beruht haben dürfte, aus Sicht des BAföG und des Unterhaltsrechts nicht zwingend ist, sondern vom Ausbildungsgang abhängt, belegen die Ausführungen von Liceni-Kierstein, Bachelor- und Masterstudium - einheitlicher Ausbildungsgang oder Doppelstudium?, in: FamRZ 2011, 526 ff.; Götz, Der Anspruch auf „eine“ Ausbildung und deren Dauer - Probleme des Ausbildungsunterhalts im deutschen Recht, in: Büchler/Müller-Chen (Hrsg.), FS Schwenzer, Bern 2011, S. 575, 585 ff. Auch in der Schweiz wird die Frage, ob eine einheitliche oder zwei getrennte Ausbildungen vorliegen, je nach Studiengang beantwortet, vgl. Hausheer/Spycher, in: Hausheer/Spycher (Hrsg.), Handbuch des Unterhaltsrechts, 2. Aufl., Bern 2010, S. 415, Rn. 06.100.

4 Zur Diskussion einer Umstellung des Jurastudiums siehe etwa Schöbel, in: Einführung des Bologna-Modells in der deutschen Juristenausbildung?, in: Baldus/Finkenauer/Rüfner (Hrsg.), Bologna und das Rechtsstudium, Tübingen 2011, S. 253 ff.; Kilian, Modelle der Juristenausbildung in Europa, Bonn 2010; Zaczyk, Rechtswissenschaft oder McLaw?, Bonner Rechtsjournal Sonderausgabe 01/2008, S. 1 ff; Konzen, Bologna-Prozess und Juristenausbildung, JZ 2010, S. 241 ff.; Konzen/Schliemann, Bologna für Juristen - Gedanken zur Reform der Juristenausbildung, in: Hanau/Thau/Westermann (Hrsg.), FS Adomeit, Köln 2008, S. 343 ff.; Baldus/Finkenauer/ Rüfner, Die Bologna-Reform aus Hochschullehrersicht, in: dies (Hrsg.) Bologna und das Rechtsstudium, Tübingen 2011, S. 281 ff.; Deutscher JuristenFakultätentag (Hrsg.), Der "Bologna-Prozess" und die Juristenausbildung in Deutschland, Stuttgart 2007; Huber, Beiträge zu Juristenausbildung und Hochschulrecht, Stuttgart 2010; Fischer/Wünsch (Hrsg.), Der Bologna-Prozess an den juristischen Fakultäten, Baden-Baden 2006; Ranieri, Juristen für Europa, Münster 2006; Ischdonat, Die deutsche Juristenausbildung unter dem Einfluss des Bologna-Prozesses, Göttingen 2010.

$5 \quad$ Zuletzt Beschluss des 87. Juristenfakultätentags vom 7./8.6.2007 (DJFT 2007/I Nr. 5); Beschluss des 88. Juristenfakultätentags vom 22./23.5.2008 (DJFT 2008/I); Beschluss des 90. Juristenfakultätentags vom 3./4.6. 2010 (DJFT 2010/I); dazu http://www.djft.de. 
penkötter und der Bundesrechtsanwaltskammer) ab. ${ }^{6}$ Die politische Diskussion mündete schließlich in den Beschluss der Justizministerkonferenz der Länder vom 18./19. Mai 2011, an der derzeit bestehenden Studiengangs- bzw. Ausbildungsorganisation festzuhalten und damit als Berufseingangsvoraussetzung weiterhin den Abschluss des Ersten und Zweiten juristischen Staatsexamens zu verlangen. ${ }^{7}$ Nach derzeitigem Stand wird es im Fach Jura also beim Staatsexamen als Regelabschluss bleiben.

Es ist den Fakultäten aber unbenommen, auch Bachelor- und/oder Masterstudiengänge anzubieten. Die Absolventen dieser Studiengänge können solche Abschlüsse als Zusatzqualifikation zum Staatsexamen erwerben (so etwa das Modell der Martin-Luther-Universität Halle Wittenberg). ${ }^{8}$ Der Bachelor- und Masterabschluss kann aber auch als alleinige Qualifikation am Ende des Jurastudiums stehen (Universität Mannheim, Universität Siegen, TU Dresden, Hanse Law School), wenn die Absolventen nicht vorhaben, in Deutschland einen der klassischen juristischen Berufe zu ergreifen. ${ }^{9}$ Ein Mischmodell findet sich an manchen Fakultäten, die als Studienziel und Regelabschluss das Staatsexamen anbieten und den Bachelor ohne oder mit sehr geringem Zusatzaufwand als Dreingabe offerieren (so die Bucerius Law School ${ }^{10}$ ), indem sie die Studienleistungen, die man auch für das Staatsexamen auf sich nehmen muss, mit Kreditpunkten versehen und dafür den Bachelor verleihen. Schließlich finden sich zahlreiche Masterprogramme, die sich als Aufbaustudiengänge an Absolventen ausländischer Hochschulen richten. Insgesamt sind im Fach Rechtswissenschaften 271 Bachelor- und Masterstudiengänge

6 Diese sind anschaulich beschrieben auf der Homepage des Kollegen Diederich Eckardt, http://www.uni-trier.de/index.php?id=6686 sowie bei Jeep, Die Reform der Juristenausbildung nach dem Koalitionsvertrag 2009, Recht und Politik 2010, S. 71 ff.; Goll, Das »Stuttgarter Modell« der Juristenausbildung, ZRP 2007, S. 190 ff.; Schöbel, Die Erste Juristische Staatsprüfung - Letzte Bastion im „Bologna-Sturm“?, JA 2011, S. 161 ff.; siehe auch die gegensätzlichen Referate von Müller-Piepenkötter und Huber, 16. Deutscher Verwaltungsgerichtstag 2010, Stuttgart 2011, S. 121 ff. sowie Huber, Zwischen Konsolidierung und Dauerreform - Das Drama der deutschen Juristenausbildung, ZRP 2007, S. $188 \mathrm{ff}$.

7 Zu finden unter http://www.justiz.de/justizministerkonferenz/index.php.

8 Dazu etwa Sethe, Spezialisierte Masterstudiengänge am Beispiel des Wirtschaftsrechtlichen Studiengangs der Juristischen Fakultät der Martin-Luther-Universität Halle-Wittenberg, in: Fischer/Wünsch (Fn. 4), S. 67 ff.

9 Andere Staaten eröffnen dagegen mit einem Masterabschluss den Zugang zur Rechtsanwaltsprüfung, so dass über den Umweg einer Zulassung in einem anderen EU-Staat und Nutzung der Niederlassungsfreiheit dieser Beruf auch in Deutschland ausgeübt werden kann.

10 „Die Bucerius Law School vergibt als zusätzlichen Abschluss zur Ersten Prüfung (das juristische Staatsexamen) den Grad eines Bachelor of Laws (LL.B.). Der LL.B. wird nicht aufgrund einer Abschlussprüfung verliehen, sondern setzt sich aus den Studienleistungen während der ersten zehn Trimester zusammen“, http://bewerberportal.law-school.de/dasbucerius-jurastudium/abschluesse.html. 
zu finden. Hiervon sind 179 Master-/Magisterstudiengänge, die in den meisten Fällen interdisziplinär anlegt sind. ${ }^{11}$

\section{Schweiz}

Die Schweiz hat sich entschlossen, alle Studiengänge auf das Bologna-System umzustellen. Um der föderalistischen Struktur der Schweiz Rechnung zu tragen, bedurfte es einer Einbindung aller für die universitäre Bildung zuständigen staatlichen Stellen. Die Schweizerische Universitätskonferenz (SUK), die ein gemeinsames Organ von Bund und Kantonen ${ }^{12}$ ist, erließ am 4. Dezember 2003 die »Richtlinien für die koordinierte Erneuerung der Lehre an den universitären Hochschulen der Schweiz im Rahmen des Bologna-Prozesses« (Bologna-Richtlinien). ${ }^{13}$ Sie waren von der Rektorenkonferenz der Schweizer Universitäten (CRUS) entworfen worden. In ihnen finden sich einheitliche Vorgaben über die Einführung der gestuften Studiengänge (dreijähriger Bachelor und eineinhalb bis zweijähriger Master) und das Kreditpunktesystem, über die Zulassung zum Masterstudium, über die einheitliche Benennung der Abschlüsse sowie über den Vollzug der Reformen. Die Bologna-Richtlinien werden durch Empfehlungen der CRUS konkretisiert.

Die Fächer Wirtschaftswissenschaften und Jura waren die ersten, die die Reform umgesetzt haben. Bereits seit dem Jahr 2001 bieten Luzern und St. Gallen das Fach Jura im Bologna-System an (im Fall der Luzerner Fakultät erfolgte dies gleich von Beginn ihres Bestehens an). ${ }^{14}$ Seit dem Wintersemester 2006/07 sind alle Studiengänge der Schweizer Universitäten und Fachhochschulen auf Bachelor und Master umgestellt. ${ }^{15}$ Auch in der Schweiz fanden und finden sich viele kritische Stimmen zum Bologna-System; bemängelt werden Inhalt und Auswirkungen der Reform, aber auch der Umstand, dass der Bund in die kantonale Hoheit über das Hochschulwesen eingegriffen habe. ${ }^{16}$

11 Hochschulrektorenkonferenz, Statistische Daten zu Bachelor- und Masterstudiengängen, Wintersemester 2011/2012, 2011, S. 9 f.

12 Art. 2 und 4 Abs. 1 der Vereinbarung vom 14. Dezember 2000 zwischen dem Bund und den Universitätskantonen über die Zusammenarbeit im universitären Hochschulbereich, AS 2001 67, SR 414.205, http://www.admin.ch/ch/d/as/2001/67.pdf.

13 Richtlinien der Schweizerischen Universitätskonferenz für die koordinierte Erneuerung der Lehre an den universitären Hochschulen der Schweiz im Rahmen des Bologna-Prozesses (Bologna-Richtlinien) vom 4. Dezember 2003, SR 414.205.1, http://www.admin.ch/ch/d/as /2004/3003.pdf.

14 Schmid, Studienberatung und Bologna-System - Ein Dankesbrief, in: Ackermann/Bommer (Hrsg.), Liber Amicorum für Dr. Martin Vonplon, Zürich 2009, S. 289, 290.

15 Staatssekretariat für Bildung und Forschung SBF, Der Bologna-Prozess in der Schweiz, http://www.sbf.admin.ch/htm/themen/uni/bologna_de.html.

16 Auer, La déclaration de Bologne et le fédéralisme universitaire en Suisse, AJP 2004, S. 712 ff.; Jaag, Bologna-Reform - Auswirkungen auf die juristische Ausbildung und Praxis, SJZ 103 (2007), S. 565 ff. 
Die Umsetzung der Reform auf universitärer Stufe erfolgte durch eigene Richtlinien und Empfehlungen der jeweiligen Universitäten, die für die Fakultäten verbindlich sind, welche ihrerseits Rahmen- ${ }^{17}$ und Studienordnungen ${ }^{18}$ ausarbeiteten. ${ }^{19}$

\section{Prüfungen}

1. Studienaufbau sowie Anzahl und Umfang der Prüfungsleistungen im Lizenziat

Das Lizenziatsstudium der rechtswissenschaftlichen Fakultät der Universität Zürich war in zwei Abschnitte unterteilt. ${ }^{20}$ In den ersten drei Semestern wurden die Grundlagen des Faches vermittelt. Es schloss eine als Lizenziat Teil I bezeichnete Prüfung an, die aus fünf Klausuren à drei Stunden in den Fächern Römisches Recht, Privatrecht I, Öffentliches Recht I, Strafrecht I und Wirtschaftswissenschaften bestand.

Das Hauptstudium, für das die Studienordnung eine Studienzeit von mindestens vier weiteren Semestern vorsah, endete mit dem Lizenziat Teil II. Dieses bestand aus drei Klausuren à fünf Stunden und sechs mündlichen Einzelprüfungen von 15 Minuten Dauer. Diese neun Prüfungen mussten in den Fächern Rechtsgeschichte, Privatrecht II, Handels- und Wirtschaftsrecht, Zivilprozess-, Schuldbetreibungsund Konkursrecht (SchKG), Strafrecht II, Öffentliches Recht II sowie drei Wahlfächern erbracht werden. Die Kandidaten konnten weitgehend wählen, in welchen Fächern sie die schriftliche und in welchen sie die mündliche Prüfung ablegen, wobei Wahlfächer immer nur mündlich geprüft wurden. Die Endnote setzte sich aus dem Durchschnitt aller Einzelnoten des ersten und des zweiten Teils zusammen, wobei die Klausuren des zweiten Teils doppelt zählten, alle anderen Prüfungen dagegen einfach.

17 So in Zürich: Rahmenordnung für das Studium in den Bachelor- und Master-Studiengängen an der Rechtswissenschaftlichen Fakultät der Universität Zürich vom 24.10.2005 i.d.F. vom 25.6.2007 (RO; LS 415.415.1).

18 In Zürich: Studienordnung Bachelor of Law (B Law) vom 8.2.2006 i.d.F. vom 20.6.2007 (StudO B Law); Studienordnung Master of Law (M Law) vom 13.12.2006 (StudO M Law). Zusätzlich wurde für Studierende anderer Fakultäten, welche Recht im Nebenfach studieren, eine Studienordnung Recht als Nebenfach auf Bachelorstufe vom 8.2.2006 i.d.F. vom 20.6.2007 erlassen, vgl. die Homepage der Rechtswissenschaftlichen Fakultät: http://www. ius.uzh.ch/rsjur.html.

19 Ausführlich dazu Jaag, SJZ 103 (2007), S. 565 ff.; Büchler/Wohlers, Bologna in der Schweiz - Zur Umgestaltung der juristischen Studiengänge an der Universität Zürich, in: ZEuP 2008, S. $110 \mathrm{ff}$.

20 Zum Folgenden $\S \S 10$ ff., 14 ff. der Promotionsordnung der Rechtswissenschaftlichen Fakultät der Universität Zürich vom 30. August 1994 (415.413), OS 56, 634. 


\section{Studienaufbau alt}

\section{Klausuren à 5 Stunden}

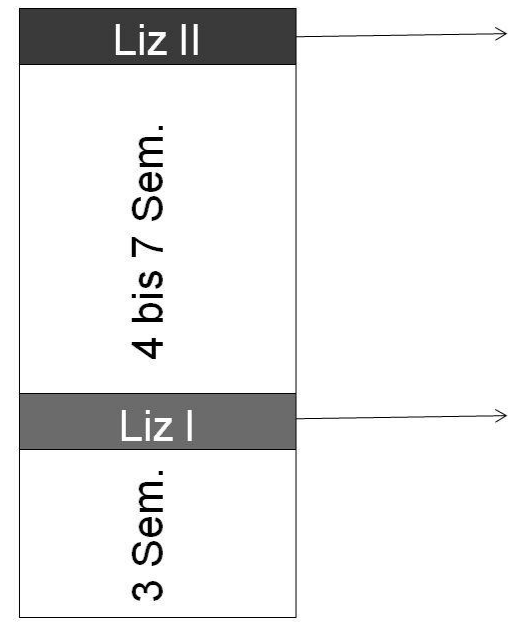

\section{6 mündliche Prüfungen à $15 \mathrm{~min}$.}

- Rechtsgeschichte

- Privatrecht II

- Handels- und Wirtschaftsrecht

- ZPO/SchKG

- Öffentliches Recht II

- Strafrecht II

- 3 Wahlfächer

\section{Klausuren à 3 Stunden}

- Römisches Recht

- Privatrecht I

- Öffentliches Recht I

- Strafrecht I

- Wirtschaftswissenschaften

Die Notenskala sah folgende Prädikate vor:

- über 5,6 summa cum laude (vorzüglich)

- über 5 bis 5,6 magna cum laude (sehr gut)

- über 4,4 bis 5 cum laude (gut)

- 4 bis 4,4 rite (genügend).

2. Studienaufbau sowie Anzahl und Umfang der Prüfungsleistungen im BolognaSystem

Seit dem Wintersemester 2006/07 bietet die Rechtswissenschaftliche Fakultät der Universität Zürich ein gestuftes Studium aus einem sechssemestrigen Bachelor of Law (B Law) und einem drei- bis viersemestrigen Master of Law (M Law) an. ${ }^{21}$ Der Pflicht- und Wahlfachstoff wurde modularisiert. An die Stelle der früheren Abschlussprüfungen treten nun Modulprüfungen am Ende jedes Semesters. Bei Erreichen von 180 ECTS Credits verleiht die Fakultät den akademischen Grad eines

21 Vgl. zum Folgenden die StudO B Law und StudO M Law (Fn. 18). 
Bachelor, bei Erreichen von weiteren 90 ECTS Credits $^{22}$ dann den Grad eines Master. Die Notenskala wurde gegenüber dem Lizenziat leicht verändert, da die Note »summa cum laude « jetzt bereits ab 5,5 vergeben wird.

Der Studiengang B Law gliedert sich in eine einjährige Assessmentstufe und eine zweijährige Aufbaustufe. In der Assessmentstufe erbringen die Studierenden den Nachweis ihrer grundsätzlichen Eignung für das Studium der Rechtswissenschaft, indem sie die im ersten Studienjahr vermittelten Grundkompetenzen im Privatrecht, Strafrecht und Öffentlichen Recht, in der Rechtsgeschichte, sowie in einem Grundlagenfach und in Wirtschaftswissenschaften nachweisen. Sie müssen drei Klausuren à zwei Stunden und fünf Klausuren à drei Stunden Bearbeitungszeit bestehen sowie eine Fallbearbeitung ${ }^{23}$ vorlegen.

In der Aufbaustufe erfolgt eine Vertiefung in den zentralen Rechtsgebieten. Die Studierenden können in diesem Studienabschnitt bereits Schwerpunkte setzen, da sie Wahlpflichtmodule wählen müssen. ${ }^{24}$ Zudem können insgesamt 6 ECTS Credits in Modulen aus anderen Fakultäten erworben werden. Um den B Law erfolgreich zu bestehen, müssen in der Aufbaustufe 21 Modulleistungen bestanden werden, nämlich 16 Klausuren oder mündliche Prüfungen (je nach Zahl der Teilnehmer der Prüfung vom Dozenten festgelegt), zwei Fallbearbeitungen, zwei Seminare und ein Wahlmodul. In der Praxis werden kaum mündliche Prüfungen in der Aufbaustufe angeboten, da aufgrund der hohen Anzahl an Prüflingen eine schriftliche Prüfung vom Aufwand her für den Prüfer effektiver ist. Daher besteht die überwiegende Zahl der Modulleistungen aus Klausuren.

Da die klassischen juristischen Berufe das Bestehen der Rechtsanwaltsprüfung erfordern und diese wiederum voraussetzt, dass man einen Masterabschluss vorweisen kann, absolvieren nahezu alle Studierenden, die den Bachelor bestanden haben, auch den Masterstudiengang. Dies dürfte sicherlich auch daran liegen, dass es bislang keine eigenständigen Berufssparten für Bachelorabsolventen in Rechtswissenschaften gibt.

Die Rechtswissenschaftliche Fakultät bietet vier Masterstudiengänge mit verschiedenen Schwerpunkten an:

- Master of Law UZH

- Master of Law UZH (Legal Practice)

- Master of Law UZH (Business Law)

- Master of Law UZH (Public Law)

22 Bzw. 120 ECTS Credits, wenn man einen der von Universität Zürich und einer ihrer Partneruniversitäten angebotenen Doppelmaster absolviert.

23 Dies entspricht etwa der Hausarbeit im ,kleinen Schein“ in Deutschland.

24 Bei der jüngst beschlossenen und ab Herbstsemester 2013 in Kraft tretenden Reform des B Law, mit der vorhandene Kinderkrankheiten ausgebessert werden, wurde die Zahl der Wahlfächer deutlich reduziert und die Aufmerksamkeit stärker auf den Pflichtstoff gelenkt, da im Master noch genügend Spezialisierungsmöglichkeiten offen stehen. 
Um eines der Masterprogramme erfolgreich zu bestehen, müssen zehn Modulprüfungen im Umfang von 60 ECTS Credits bestanden werden, wobei die Modulleistungen aus Klausuren mit drei Stunden Bearbeitungszeit bestehen, es sei denn, der Dozent entschließt sich, aufgrund der geringen Zahl von Prüflingen eine mündliche Prüfung anzubieten. Weitere 30 ECTS Credits müssen in Form einer Masterarbeit erbracht werden, wobei diese auch auf zwei Einzelarbeiten zu 12 und 18 ECTS Credits aufgeteilt werden kann. ${ }^{25}$ Im Ergebnis gestaltet sich das Studium nach Bologna wie folgt:

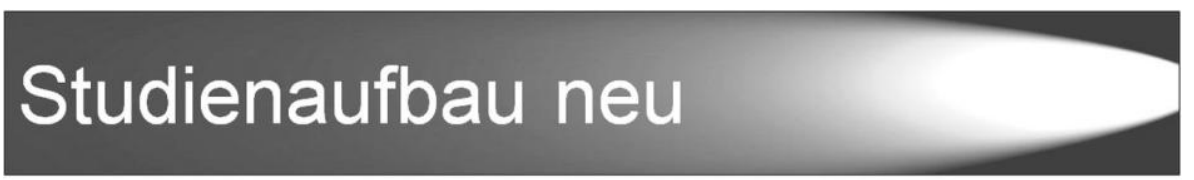

\begin{tabular}{|c|c|}
\hline $\begin{array}{l}\text { Master } \\
3 \text { Sem. } \\
\text { (MLaw) }\end{array}$ & $\begin{array}{l}\text { Masterarbeit(en) (30 ECTS) } \\
10 \text { Modulprüfungen (60 ECTS) } \\
\text { - Klausuren à } 3 \text { Stunden } \\
\text { • z.T. ersetzt durch mündl. Prüfungen }\end{array}$ \\
\hline $\begin{array}{l}\text { Aufbaustufe } \\
4 \text { Sem. } \\
\text { (BLaw) }\end{array}$ & $\begin{array}{l}21 \text { Modulprüfungen (120 ECTS) } \\
\text { - } 16 \text { Klausuren oder mündl. Prüfungen } \\
\text {-2 Fallbearbeitungen } \\
\text { - } 2 \text { Seminare } \\
\text {-1 Wahlmodul }\end{array}$ \\
\hline $\begin{array}{c}\text { Assessment } \\
2 \text { Sem. }\end{array}$ & $\begin{array}{l}9 \text { Modulprüfungen (60 ECTS) } \\
\text { - } 3 \text { Klausuren à } 2 \text { Std. } \\
\text { - } 5 \text { Klausuren à } 3 \text { Std. } \\
\text { - } 1 \text { Fallbearbeitung }\end{array}$ \\
\hline
\end{tabular}

25 Nach Drucklegung des Beitrags wurde diese Vorgabe geändert. Der Umfang der Masterarbeit(en) wurde von 30 auf 18 ECTS Credits reduziert, wobei die Studierenden nun die Wahl haben, ob sie die Differenz von 12 ECTS Credits durch Klausuren oder eine weitere Masterarbeit abdecken. 
3. Folgen des Systemwechsels für das Prüfungsgeschehen

a) Prüfungsbelastung

Der Wechsel zum Bologna-System hat enorme Folgen für die Lehrstühle, denn es kam zu einer massiven Zunahme von Prüfungen und von Seminararbeiten. Die Korrektur der Masterarbeiten bedeutet eine erhebliche zusätzliche Belastung. Diese durch Bologna bedingte Entwicklung wird noch dadurch verstärkt, dass die Zahl der Studierenden in den letzten Jahren erheblich zugenommen hat. Als ausschließlich Lizenziatsprüfungen abzunehmen waren, bewegte sich deren Zahl bei durchschnittlich 2.000 pro Semester. ${ }^{26}$ Wie die folgende Tabelle zeigt, hat die Umstellung auf das Bologna-System zu einem allmählichen Ansteigen und letztlich zu einer Verfünffachung der Gesamtzahl der Prüfungen geführt:

\begin{tabular}{|l|r|r|r|}
\hline Semester & \multicolumn{1}{|c|}{ B Law } & \multicolumn{1}{|c|}{ M Law } & Lizenziat (auslaufend) \\
\hline HS 2009 & 6.850 & 670 & 1.800 \\
\hline FS 2010 & 7.870 & 710 & 2.000 \\
\hline HS 2010 & 7.700 & 1.300 & 1.500 \\
\hline FS 2011 & 8.000 & 1.370 & 1.600 \\
\hline HS 2011 & 8.140 & 1.810 & 400 \\
\hline
\end{tabular}

Da die Universitätsleitung frühzeitig erkannte, dass der Systemwechsel den Prüfungs- und Betreuungsaufwand für den Lehrkörper erheblich erhöht, stockte sie die Zahl der Lehrstühle um $25 \%$ auf. Nötig war auch eine Verbesserung der Finanzausstattung der Fakultät, denn pro Semester fallen ca. 300.000 Fr. für Prüfungsaufsicht und die Korrekturen an. Da die Fakultät keine ausreichend großen Räume für derart viele Prüfungen hat, finden diese nun in der Zürcher Messe statt, deren Miete 50.000 Fr. beträgt. Um in der Messe angemessene Prüfungsbedingungen zu schaffen, bedurfte es zudem der Anschaffung von Tischen und Stühlen, was rund 250.000 Fr. kostete.

b) Noteninflation

Der Systemwechsel hat sich auch deutlich in den erzielten Ergebnissen der Prüfung niedergeschlagen, wie die nachfolgende Tabelle ${ }^{27}$ mit den Notendurchschnitten der letzten Jahre zeigt:

26 Alle nachfolgenden Angaben verdanke ich Dr. Urs Leemann, dem Leiter Lehre und Organisation der Rechtswissenschaftlichen Fakultät der Universität Zürich, dem ich nochmals herzlich für seine Mühen danke.

27 Vgl. Fn. 26. 


\begin{tabular}{|l|r|r|r|}
\hline Notenstufen & \multicolumn{1}{|c|}{ Lizenziat } & \multicolumn{1}{|c|}{ B Law } & \multicolumn{2}{c|}{ M Law } \\
\hline summa cum laude & $1,27 \%$ & $3,39 \%$ & FS 2011 - HS 2011 \\
\hline magna cum laude & $17,18 \%$ & $18,32 \%$ & $51,40 \%$ \\
\hline cum laude & $59,20 \%$ & $55,48 \%$ & $34,88 \%$ \\
\hline rite & $19,69 \%$ & $22,81 \%$ & $0,60 \%$ \\
\hline nicht bestanden & $2,67 \%$ & & \\
\hline
\end{tabular}

Die Notenstufe »rite«, die vor der Reform ungefähr ein Fünftel ausmachte, kommt im Master so gut wie nicht mehr vor. Die Zahl der Abschlüsse mit »summa cum laude« verzehnfachte sich. Im Ergebnis hat sich die Notenskala vom Lizenziat zum Master um eine ganze Notenstufe nach oben verschoben.

c) Vergleich mit den Ergebnissen im ersten Staatsexamen

Der Vergleich mit den Ergebnissen des deutschen Ersten juristischen Staatsexamens (2001-2006) ${ }^{28}$ offenbart, dass die deutschen Prüfungsergebnisse im Vergleich zum Lizenziat nochmals deutlich strenger ausfallen.

\begin{tabular}{|l|r|r|}
\hline Notenstufen & \multicolumn{1}{|c|}{ Lizenziat } & \multicolumn{1}{c|}{ Staatsexamen } \\
\hline summa cum laude / sehr gut & $1,27 \%$ & $0,18 \%$ \\
\hline magna cum laude / gut + vollbefriedigend & $17,18 \%$ & $15,04 \%$ \\
\hline cum laude / befriedigend & $59,20 \%$ & $26,73 \%$ \\
\hline rite / ausreichend & $19,69 \%$ & $30,30 \%$ \\
\hline nicht bestanden & $2,67 \%$ & $27,57 \%$ \\
\hline
\end{tabular}

Das Fach Rechtswissenschaften hat in Deutschland ohnehin den Ruf, über Jahrzehnte konstant niedrige Noten zu vergeben. Die Notenskala, die von null bis 18 Punkten reicht, wird selten ausgeschöpft und der Durchschnitt der Examen liegt regelmäßig zwischen fünf und sechs Punkten. Dass die Juristen sich damit deutlich von anderen Fächern abheben, verdeutlicht folgende Tabelle zur Häufigkeit der Vergabe der Bestnote im jeweiligen $\mathrm{Fach}^{29}$ :

28 Berechnet nach der Statistik von Karger, Rekonstruktion des Rechtsunterrichts am Beispiel des materiellen Strafrechts, Baden-Baden 2009, S. 18.

29 Spiegel-Online vom 20.4.2007 http://www.spiegel.de/unispiegel/studium/0,1518,478364, 00.html. Vergleichbare Zahlen aus der Schweiz standen nicht zur Verfügung. Vgl. umfassend auch den Arbeitsbericht des Wissenschaftsrates, Prüfungsnoten an Hochschulen im Prüfungsjahr 2010, Drs. 2627-12. 


\begin{tabular}{|l|r|}
\hline Fach & Note $»$ sehr gut $«$ oder $»$ mit Auszeichnung $«$ \\
\hline Jura $($ Note $» g u t «+~ »$ sehr gut $\ll)$ & $3,5 \%$ \\
\hline Bau-Ingenieurwesen & $4,7 \%$ \\
\hline Pharmazie & $5,5 \%$ \\
\hline Wirtschaftswissenschaften & $7,2 \%$ \\
\hline Humanmedizin & $8,9 \%$ \\
\hline Architektur & $14,9 \%$ \\
\hline Elektrotechnik & $17,6 \%$ \\
\hline Maschinenbau & $22,1 \%$ \\
\hline Erziehungswissenschaften & $33,4 \%$ \\
\hline Informatik & $35,2 \%$ \\
\hline Germanistik & $36,4 \%$ \\
\hline Psychologie & $51,8 \%$ \\
\hline Biologie & $60,9 \%$ \\
\hline
\end{tabular}

d) Mögliche Gründe für die Noteninflation

Die Möglichkeit, im Bologna-System Prüfungen abzuschichten, begünstigt auch schwächere Kandidaten. Das Bologna-System ging mit einer Ausweitung der Kurse auf der Masterstufe einher. Anders als bei Pflichtveranstaltungen, die einen ganzen Jahrgang erfassen, hat man es hier oft mit »kleinteiligen« Kursen zu tun, die keine gute Vergleichbarkeit der Kandidaten ermöglichen, was mitunter zu einer zu guten Benotung führt. Vielen Studierenden fallen Masterarbeiten leichter als Abschlussklausuren. ${ }^{30}$ Weiterhin ist festzustellen, dass bei Wahlmöglichkeiten, wie sie vor allem im Masterstudium möglich sind, als schwer empfundene Fächer abgewählt und unbeliebte Dozenten gemieden werden. Zur Noteninflation beigetragen hat schließlich die Verschiebung der Prädikatsstufe »summa cum laude«, die früher ab 5,6 und jetzt ab 5,5 vergeben wird.

e) Konsequenzen der Noteninflation

Im Zuge der Recherchen für diesen Beitrag habe ich mit drei Personalverantwortlichen aus Zürcher Großkanzleien gesprochen, die sich jeweils darüber beklagten,

30 Dieses Phänomen lässt sich auch in Deutschland bei den Hausarbeiten im Rahmen des Schwerpunktstudiums beobachten. Auch einige deutsche Kanzleien bemängeln daher, dass seit Einführung des Schwerpunktbereichsstudiums die Aussagekraft der Examensgesamtnote abgenommen habe, weshalb man zunehmend auf die Noten allein des staatlichen Teils abstellt. 
dass sie als Arbeitgeber nun nicht mehr wüssten, wer wirklich herausragend sei. Diese negative Folge ist in der Schweiz nochmals spürbarer, da die Schweizer Notenskala weniger Differenzierungen als die deutsche kennt.

Wenn aber nahezu zwei Drittel der Absolventen ein »gut« oder »sehr gut« als Abschlussnote mitbringt (s.o. III. 3 b) ${ }^{31}$, werden andere Faktoren beim Einstellungsgespräch wichtiger (z.B. soziale Herkunft, Geschlecht). Wenn die Noten weniger aussagekräftig sind, suchen Studierende frühzeitig nach anderen Qualifikationen, mit denen sie sich auszeichnen können; es besteht damit ein faktischer Zwang zur Zusatzqualifikation (»Nebenjobs«), was wiederum eine Studienzeitverlängerung zur Folge hat. Die Noteninflation erschwert auch den sozialen Aufstieg, da sich Absolventen aus Bildungshaushalten noch leichter durchsetzen als bisher. Schließlich besteht die Gefahr, dass der »Universaljurist« verloren geht, da die Fächer oft nur nach der Benotung ausgesucht werden.

\section{Schlussfolgerungen}

- Es wäre zu früh, bereits jetzt eine generelle Aussage zu der Frage zu treffen, ob das Bologna-System gegenüber dem Lizenziat vor- oder nachteilig ist. Die Zürcher Fakultät ist gerade dabei, erkannte Schwächen der Bologna-Reform zu beheben. Dazu gehört einerseits, die Zahl der Wahlmöglichkeiten im Bachelor zu reduzieren und eine Vertiefung der klassischen Pflichtfächer vorzuschreiben, um eine solide Grundausbildung im Fach Jura zu gewährleisten. Im Masterstudium wurde bereits eine erste Änderung umgesetzt: Die Masterarbeit fließt regelmäßig nur noch im Umfang von 18 statt 30 ECTS Credits in die Note ein.

- Derzeit stehen Bachelorabsolventen in der Schweiz noch keine eigenständigen juristischen Berufsfelder offen. Aufgrund der Regelung, dass die Zulassung zur Rechtsanwaltsprüfung einen Masterabschluss voraussetzt, wird der Master in der Schweiz der Regelabschluss sein.

- Das Bologna-System geht mit einer Vervielfachung von Prüfungsleistungen einher. Für Deutschland lässt sich daraus die Schlussfolgerung ziehen, dass ein Wechsel vom Modell des Staatsexamens zum B Law und M Law nicht ratsam erscheint, da die Finanznot der Fakultäten so groß ist, dass sich die Studienbedingungen weiter verschlechtern würden. Da die Politik in den letzten Jahren

31 Die Rechtswissenschaftliche Fakultät gibt auf ihrer Homepage zusätzlich zur Abschlussnote eine Rangliste heraus, die die Top 10\%, 25\% etc. ausweist. So kann der Arbeitgeber immerhin erkennen, wie der Einzelabschluss im Vergleich zu den Ergebnissen des Gesamtjahrgangs einzuordnen ist. Anders als die Notenskala beim Lizenziat, bei der der Arbeitgeber wusste, dass ein »summa cum laude« aufgrund seiner Seltenheit die Zugehörigkeit zu den Top $1 \%$ bedeutete, gibt die Rangliste nun nur noch die Top $10 \%$ bekannt. 
nicht wirklich willens war, die Finanznot der juristischen Fakultäten zu beheben, erschiene ein Wechsel ohne definitive Finanzierungszusagen mehr als gewagt.

- Die mit dem Wechsel zum Bologna-System in Zürich zu beobachtende Noteninflation ist bedenklich. Sie benachteiligt Personen aus bildungsfernen Haushalten, die sich nicht mehr nur über Leistungen im Fach Jura auszeichnen können. Sie kann zu einer Studienzeitverlängerung führen. Arbeitgeber müssen u.U. eigene Tests einführen, um in der Masse der Bewerber mit den Noten »magna cum laude « und »summa cum laude « die wirklich guten Absolventen zu erkennen. Die Zürcher Fakultät ist sich des Problems bewusst und sucht derzeit nach Lösungen. 